\title{
TENDÊNCIA SECULAR DE CRESCIMENTO EM PRÉ-ESCOLARES, BRASIL
}

\author{
SECULAR TREND OF GROWTH OF \\ PRESCHOOL, BRAZIL
}

\author{
Viviane Gabriela Nascimento ${ }^{1}$ \\ Ciro João Bertoli ${ }^{2}$ \\ Lucia Musmê Queiroga Bertoli ${ }^{2}$ \\ Rubens Feferbaun ${ }^{3}$ \\ Luiz Carlos de Abreu ${ }^{1}$ \\ Claudio Leone ${ }^{4}$
}

Nascimento, VG et al. Tendência secular de crescimento de crianças pré-escolares, Brasil, 19972007. Rev. Bras. Cresc. e Desenv. Hum. 2010; 20(2): 199-207.

\begin{abstract}
Resumo
Introdução: a análise da distribuição temporal de eventos do processo saúde-doença é uma das estratégias de investigação mais antigas e valiosas para a epidemiologia e para a saúde pública. Objetivo: analisar a evolução do perfil antropométrico de pré-escolares, que frequentavam creches municipais da cidade de Taubaté em 1997 e 2007. Método: foram analisadas variáveis do banco de dados de 2 estudos transversais: um realizado em 1997, com 755 crianças, e outro de 2007, com 1448 crianças. Os dados analisados foram: idade da criança, sexo, peso, estatura e índice de massa corporal (IMC). A transformação dos valores antropométricos em escores Z foi realizada pelo referencial do CDC/NCHS (2000). As comparações entre os dois momentos foram feitas pelo $\chi$-quadrado e pelo teste t-Student, $(\alpha=$ 0,05). Resultados: evidenciou-se tendência de crescimento positiva entre 1997 e 2007 com um aumento significante nos valores médios de escore $Z$ de estatura em ambos os sexos, meninos: 0,04 para 0,39 e meninas: 0,05 para 0,33 , e de peso, meninos; $-0,03$ para 0,26 e meninas: 0,03 para 0,21. As médias de IMC não revelaram diferença significante (meninos de - 0,02 para 0,07 e meninas de 0,09 para 0,12). Observou-se também, entre 1997 e 2007, um aumento na prevalência de crianças emagrecidas, de 9,8 para $12,6 \%$ e de crianças com excesso de peso, de 17,0 para 20,9\%. Conclusão: os pré-escolares da cidade de Taubaté tiveram uma tendência secular de crescimento bastante significativa entre 1997 e 2007, mais de estatura do que de peso, que se acompanhou de um aumento na prevalência de crianças emagrecidas e, principalmente, com excesso de peso. Isso indica que, possivelmente, a melhoria das condições de bem estar da população, além de repercutir positivamente no crescimento estatural dos préescolares, também pode resultar na instalação de um intenso e precoce processo de transição nutricional, inclusive fora das grandes regiões metropolitanas.
\end{abstract}

Palavras-chave: crescimento, antropometria, pré-escolar, tendência secular.

\footnotetext{
Pesquisadores. Departamento de Saúde Materno-Infantil da Faculdade de Saúde Pública da Universidade de São Paulo. Disciplina de Pediatria do Departamento de Medicina da Universidade de Taubaté.

Livre docente. Departamento de Pediatria da Faculdade de Medicina da Universidade de São Paulo

Professor Titular do Departamento de Saúde Materno-Infantil da Faculdade de Saúde Pública da Universidade de São Paulo. Trabalho realizado em colaboração entre a Disciplina de Pediatria do Departamento de Medicina da Universidade de Taubaté e e Departamento de Saúde Materno-Infantil da Faculdade de Saúde Pública da Universidade de São Paulo. Av. Dr. Arnaldo, 715 - CEP 01246-904 - São Paulo - SP.

Correspondência para: Viviane Gabriela Nascimento. Departamento de Saúde Materno-Infantil. Tel.: (11) 3085-0240. Tel./Fax: (11) 3081-2451. Av. Dr. Arnaldo, 715- CEP: 01246-904. São Paulo - SP.

Todos os autores se responsabilizam pelo conteúdo apresentado no trabalho. Não há conflito de interesse. O trabalho foi aprovado pelo Comitê de Ética em Pesquisa da Universidade de Taubaté (protocolo no $02 / 05$ em 18/02/2005).
} 


\begin{abstract}
:
Introduction: the analysis of the temporal distribution of events in the health-disease process is one of the strategies of the oldest and most valuable research in epidemiology and public health. Objective: To analyze changes in the anthropometric profile of preschool children that frequented public day-care centers of Taubate, Brazil. Method: 755 children in 1997 and 1448 in 2007 were evaluated. The study variables were: age, sex, weight, stature and body mass index (BMI). The transformation of the anthropometric values in $\mathrm{Z}$ scores was carried through the reference data of the CDC/NCHS (2000). The comparisons between the two moments were made by $\chi$ squared and t-Student tests $(\alpha=0,05)$. Results: positive secular trend of growth was observed between 1997 and 2007 with a significant increase in the average values of $Z$ scores of stature in both sexes, boys: 0,04 to 0,39 and girls: 0,05 to 0,33 , and of weight, boys; $-0,03$ to 0,26 and girls: 0,03 to 0,21 . The averages of BMI showed no significant difference (boys 0,02 to 0,07 and girls 0,09 to 0,12 ). There was an increase of thinness prevalence, 9,8 to $12,6 \%$ children, and also of weight excess, 17,0 to 20,9\%, between 1997 and 2007. Conclusions: Preschool children from the city of Taubaté showed a quite significant increase in the growth trend between 1997 and 2007, more height than weight, which was accompanied by an increased prevalence of thin children and especially overweight. This indicates that possibly the improvement of the population, and impact positively on growth in stature of preschool children, can also result in the installation of an early and intense nutritional transition process, even outside the major metropolitan areas.
\end{abstract}

key words: growth; anthropometry; preschool children; secular trend.

\section{INTRODUÇÃO}

A análise da distribuição temporal de eventos do processo saúde-doença é uma das estratégias de investigação mais antigas e valiosas para a epidemiologia e para a saúde pública. Quando a análise envolve períodos prolongados de tempo, costuma-se denominá-la de análise de tendência e/ou de mudança secular ${ }^{1}$.

O crescimento e desenvolvimento físico de crianças são reconhecidos como indicadores sensíveis das condições de saúde e nutrição da população ${ }^{2}$. Fatores ambientais como condição socioeconômica, controle de doenças infecciosas através de imunização em massa, condições sanitárias, renda mínima, nível de educação, industrialização e urbanização são significantes para a contribuição da tendência secular por serem bloqueadores do potencial biológico ${ }^{3}$.

A velocidade do crescimento e a altura alcançada nas diferentes idades são fenótipos condicionados pela herança genética dos indivíduos. A realização plena desse potencial genético depende da existência de boas condições de vida em geral, de um ótimo estado nutricional, ou seja, alimenta- ção adequada e, por extensão, de um bom estado de saúde ${ }^{4}$.

A tendência secular de crescimento físico humano é uma experiência natural que evidencia a complexa interação entre genes, fisiologia e ambiente para determinação da forma e tamanho dos indivíduos de uma geração para a seguinte ${ }^{5}$. Mudanças nas dimensões corpóreas tem chamado atenção dos antropologistas ${ }^{6}$ e crianças em países desenvolvidos documentam bem esse fenômeno ${ }^{7}$.

A investigação da tendência secular do crescimento, principalmente em estatura, é considerada atualmente um importante indicador para o monitoramento de mudanças no padrão econômico, de saúde e nutrição de uma população, quando comparada aos obtidos a partir de dados exclusivamente econômicos ${ }^{(8,9)}$; por isso a utilização de dados de crescimento passou a ser uma metodologia que vem sendo proposta para caracterizar as condições de bem estar de populações, bem como a sua evolução ao longo do tempo ${ }^{10}$.

Desta maneira, o objetivo é analisar a evolução do perfil antropométrico de pré-escolares que frequentavam as creches municipais da cidade de Taubaté. 


\section{MÉTODO}

Estudo analítico com comparação dos dados de crescimento de crianças de 3 a 6 anos de idade completos, matriculadas em creches municipais da cidade de Taubaté, realizado através da análise dos dados de antropometria de dois estudos transversais realizados nos anos de 1997 e 2007.

A amostragem nos dois momentos (1997 e 2007) foi realizada por conglomerados, de maneira probabilística, a partir da listagem das creches municipais do Departamento de Educação e Cultura da Prefeitura de Taubaté, que conta atualmente com 70 creches. Em ambos os estudos as creches foram a unidade amostral, sorteadas de maneira probabilística, sequencialmente, até que se completasse o lote amostral de crianças estimado como necessário para cada sexo.

A amostra de 1997 foi calculada considerando um nível de significância de 5\%; um poder de teste de $90 \%$; de modo detectar como estatisticamente significante uma diferença de um quarto de desvio padrão entre a média de estatura do referencial utilizado e média da população de estudo. O tamanho mínimo de amostra estimado como necessário foi de 320 crianças para cada sexo.

Em 2007 o cálculo de amostra foi realizado, modificando-se apenas a diferença com o referencial, que foi reduzida para um quinto de desvio padrão, visando diminuir o intervalo de confiança da média da população estudada, o que resultou numa amostra mínima de 600 crianças para cada sexo. Em ambos os momentos o tamanho de amostra estimado foi aumentado em 15\%, em função da possibilidade de eventuais perdas.

Após o sorteio das creches a amostra final obtida em 1997 foi de 755 crianças e em 2007 foi de 1448, o que representa, em ambos os estudos, cerca de $7 \%$ do total de crianças que estavam frequentando as creches municipais da cidade de Taubaté nos dois momentos.
Em ambos os estudos a coleta de dados foi realizada da mesma maneira, ou seja, durante o ano letivo das crianças, tendo como critério de exclusão as crianças que apresentavam doenças crônicas, que pudessem interferir no crescimento da criança.

As variáveis de estudo foram: idade da criança, sexo, peso e estatura atual e índice de massa corporal (IMC). Os dados antropométricos foram coletados na própria creche, após o consentimento dos pais ou responsáveis, segundo o método de Lohman et al ${ }^{11}$. O peso da criança foi aferido utilizando a balança eletrônica marca Kratos com divisão de 10 g, e a estatura medida com estadiômetro de parede com dois metros e subdivisão em centímetros e milímetros desenvolvido por Leone et al ${ }^{12}$. As informações sobre idade e sexo das crianças foram obtidas através da ficha fornecida pelo Departamento de Educação e Cultura da Prefeitura da cidade de Taubaté.

A transformação dos valores de estatura, peso e índice de massa corporal (IMC $=$ peso $[\mathrm{kg}] /$ altura $\left.^{2}[\mathrm{~m}]\right)$ em escores $\mathrm{Z}$ para o sexo e idade foi realizada a partir dos dados do referencial proposto pelo Centers for Diseases Control (CDC)/ National Center for Health Statistics, EUA, do ano $2000^{13}$.

Para caracterização da população de estudo foi utilizada a distribuição de frequência por faixas de escores Z. As faixas adotadas foram: menor ou igual a $-1,28$ escores $Z$ (correspondente ao percentil 10, considerado como ponto de corte para risco de magreza/desnutrição) $)^{14},-1,27$ a $1,>1$ a $<2$ e $\geq 2$ escores $Z$, estes dois últimos propostos atualmente pela Organização Mundial de Saúde como risco de sobrepeso e obesidade ${ }^{15}$.

A comparação das proporções de crianças nas faixas de escores Z entre 1997 e 2007 foi feita pelo teste de $\chi$-quadrado e a comparação das médias de escore $Z$ de estatura, peso e IMC das amostras como um todo e para ambos os sexos nos dois momentos foi realizada pelo teste t de Student para dados não pareados. 
A análise estatística foi feita utilizando o software MedCalc versão 10.1.2.0.

Os estudos que geraram os dados apresentados nesta análise foram aprovados, antes de sua realização, pelo Comitê de Ética em Pesquisa da Universidade de Taubaté (CEP 02/05).

\section{RESULTADOS}

Fizeram parte da amostra de 1997, 364 $(48,2 \%)$ crianças do sexo feminino e 391 (51,8\%) masculino. Entre as meninas a média de idade foi de 66,7 meses, desvio padrão (dp) de 13,2 e mediana de 70 meses. Entre os meninos a média de idade foi de 64,5 meses, desvio padrão de 13,7, e mediana de 68 meses.

Em 2007 a amostra foi composta por 754(52,1\%) crianças do sexo feminino e $694(47,9 \%)$ do masculino. Entre as meninas a média de idade foi de 66,6 meses, com dp de 10,6 , e mediana de 67,6 meses. Para os meninos a idade média foi de 66,2 meses com dp de 10,3 e mediana de 67,0 meses.

Em 1997, os pré-escolares apresentavam escores Z médios ( \pm dp) de estatura, peso e IMC respectivamente de 0,04 ( $\pm 1,003), 0,01$ $( \pm 1,034)$ e $0,03( \pm 1,076)$, já em 2007 as médias foram $0,36( \pm 0,979), 0,24( \pm 1,072)$ e 0,09 $( \pm 1,191)$ respectivamente. Os valores de esta- tura e peso diferiram de maneira estatisticamente significante (t: 7,22; $\mathrm{p}<0,0001$ e t: 4,83; $\mathrm{p}<0,0001)$, não se observando diferença entre as médias de IMC $(\mathrm{p}>0,05)$.

A tabela 1 mostra diminuição na prevalência de crianças em risco de baixa estatura durante o período estudado, o mesmo ocorrendo quanto ao risco de peso baixo. Por outro lado o IMC evidencia uma elevação da prevlência de crianças classificáveis como em risco de magreza.

No outro extremo da distribuição observa-se um aumento da frequência de crianças mais altas e mais pesadas para sexo e idade, o que pode representar uma maior prevalência, após uma década, de crianças em risco de excesso de peso.

Na Tabela 2 observa-se que em 2007 houve um aumento significante nos valores médios de escore $\mathrm{Z}$ de estatura e de peso, tanto em meninos quanto em meninas, que, no entanto, não influenciaram as médias de IMC, que não revelou diferença estatisticamente significante entre os dois anos estudados.

Nas figuras 1, 2 e 3 observa-se que houve um deslocamento das curvas de distribuição de escores $\mathrm{Z}$ da população de pré-escolares de 2007 como um todo em direção a valores maiores quando comparados com as curvas de distribuição de 1997. Neste sentido, chama

Tabela 1: Distribuição dos pré-escolares de Taubaté de acordo com seus escores Z de estatura (E), peso (P) e índice de massa corpórea (IMC), em 1997 e 2007.

\begin{tabular}{|c|c|c|c|c|c|c|}
\hline & $\begin{array}{c}\text { E 1997* } \\
\text { n(\%) }\end{array}$ & $\begin{array}{c}\text { E } 2007 * \\
\text { n(\%) }\end{array}$ & $\begin{array}{c}\text { P 1997** } \\
\text { n(\%) }\end{array}$ & $\begin{array}{c}\text { P 2007** } \\
\text { n(\%) }\end{array}$ & $\begin{array}{c}\text { IMC1997*** } \\
\mathrm{n}(\%)\end{array}$ & $\begin{array}{c}\text { IMC } 2007 * * * \\
\mathbf{n}(\%)\end{array}$ \\
\hline$\leq-1,28$ & $65(8,6)$ & $57(3,9)$ & $79(10,5)$ & $101(6,9)$ & $74(9,8)$ & $183(12,6)$ \\
\hline$>-1,28 a+1$ & $565(74,8)$ & $1026(70,9)$ & $552(73,1)$ & $1019(70,4)$ & $553(73,2)$ & $962(66,5)$ \\
\hline$>1 \mathrm{a}<2$ & $103(13,7)$ & $299(20,6)$ & $97(12,8)$ & $231(16,0)$ & $101(13,4)$ & $217(15,0)$ \\
\hline$\geq 2$ & $22(2,9)$ & $66(4,6)$ & $27(3,6)$ & $97(6,7)$ & $27(3,6)$ & $86(5,9)$ \\
\hline Total & $755(100,0)$ & $1448(100,0)$ & $755(100,0)$ & $1448(100,0)$ & $755(100,0)$ & $1448(100,0)$ \\
\hline
\end{tabular}


Figura 1: Distribuição de frequência de pré-escolares de creches municipais segundo o escore Z de estatura para idade e sexo. Taubaté, SP, 1997 e 2007.

CDC/NCHS 2000

Taubate 1997

Taubate 2007

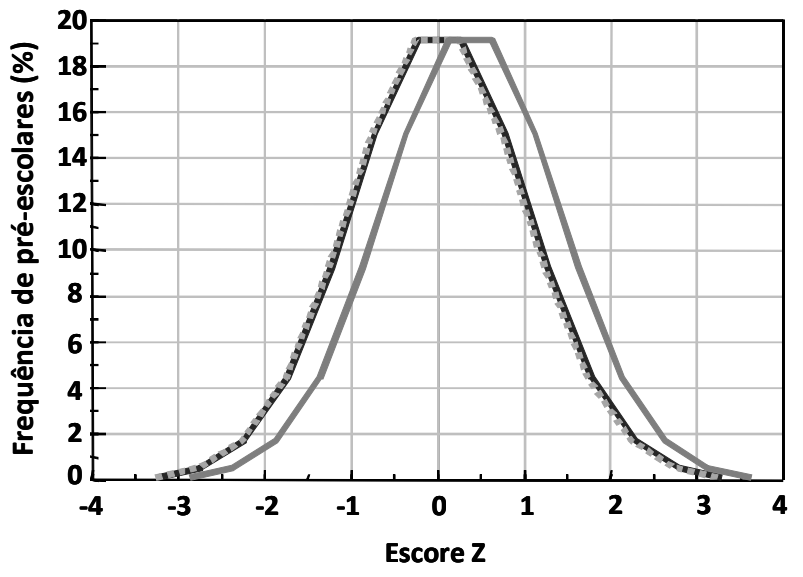

Figura 2: Distribuição de frequência de pré-escolares de creches municipais segundo o escore Z de peso para idade e sexo. Taubaté, SP, 1997 e 2007.

CDC/NCHS 2000

Taubate 1997

Taubate 2007

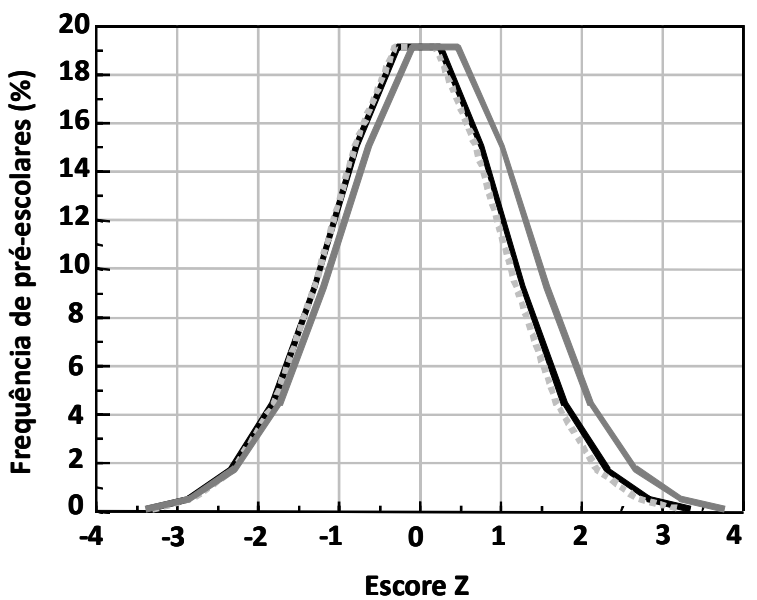

Figura 3: Distribuição de frequência de pré-escolares de creches municipais segundo o escore Z de índice de massa corpórea para a idade e sexo, Taubaté, SP, em 1997 e 2007

CDC/NCHS 2000

Taubate 1997

Taubate 2007

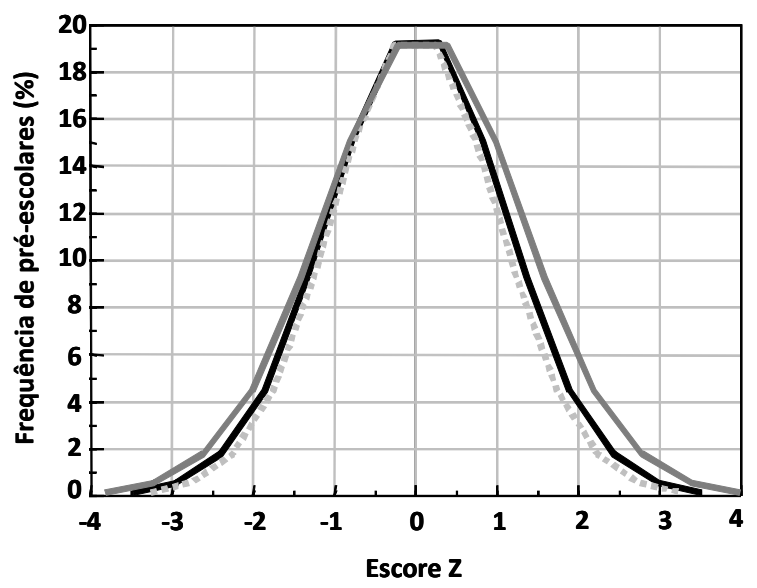


Tabela 2: Escore Z de estatura, peso e índice de massa corporal dos pré-escolares de Taubaté, segundo sexo, em 1997 e 2007.

\begin{tabular}{|c|c|c|c|c|}
\hline \multirow[t]{2}{*}{ Escore Z } & \multicolumn{2}{|c|}{ Masculino* } & \multicolumn{2}{|c|}{ Feminino** } \\
\hline & 1997 média ( \pm dp) & 2007 média ( \pm dp) & 1997 média ( \pm dp) & 2007 média $( \pm d p)$ \\
\hline Estatura/idade $^{1}$ & $0,04( \pm 1,02)$ & $0,39( \pm 0,97)$ & $0,05( \pm 1,01)$ & $0,33( \pm 0,98)$ \\
\hline Peso/idade ${ }^{2}$ & $-0,03( \pm 1,01)$ & $0,26( \pm 1,05)$ & $0,03( \pm 1,09)$ & $0,21( \pm 1,09)$ \\
\hline IMC $^{3}$ & $-0,02( \pm 1,05)$ & $0,07( \pm 1,19)$ & $0,09( \pm 1,10)$ & $0,12( \pm 1,19)$ \\
\hline
\end{tabular}

também a atenção, que em 1997 os pré-escolares já apresentavam uma curva de distribuição de frequências praticamente idêntica à do referencial adotado.

Por outro lado a figura 3, do IMC, mostra também um pequeno deslocamento em direção a escores menores de IMC, confirmando um aumento da prevalência também de crianças magras, como observado na tabela 1.

\section{DISCUSSÃO}

Os dados apresentados neste estudo mostram que as crianças de 2007 tiveram aumento de estatura, alcançando uma média 1,4cm maior do que a de 1997. Em relação ao peso, observou-se aumento em média de 500$600 \mathrm{~g}$ em 2007, confirmando uma tendência secular de crescimento positiva.

Os aumentos observados em e statura comumente têm sido atribuídos a melhorias nas condições sanitárias, econômicas e sociais. Entre as correntes que tentam explicar o fenômeno, destaca-se a que sugere importante contribuição do meio ambiente, por exemplo, com aumento na disponibilidade de alimentos e um maior acesso aos serviços essenciais ${ }^{9}$.

Estudo realizado para avaliar a tendência secular de estatura, peso e a relação peso e estatura, em escolares de Paulínia, São Paulo, Brasil, entre 1979/80 e 1993/94, e observou que a média de estatura e peso foi sempre superior às do estudo anterior, porém para a relação peso para estatura as médias dos dois estudos foram muito semelhantes em ambos os sexos, não mostrando modificações. O estudo conclui que houve uma tendência secular positiva de crescimento da estatura e do peso, que seria atribuível principalmente ao desenvolvimento econômico, com melhorias de habitação, no saneamento básico, na educação e na área de saúde, ocorrido no período de estudo ${ }^{16}$.

Durante o período do atual estudo, a cidade de Taubaté, de acordo com a Fundação SEADE, mostrou um PIB per capita em 1999 de $\mathrm{R} \$ 10.549,62$ e em 2006 de $\mathrm{R} \$$ 19.872,64, uma Taxa de Mortalidade Infantil em 1997 de $20,68 \%$ e 2007 de $14,25 \%$, ou seja, houve uma diminuição de 6,4\%o nascidos vivos, um Índice de Desenvolvimento Humano Municipal (IDHM) que na década de 90 foi de 0,797 e que em 2000 passou para 0,837. Em 2003 a cidade atingiu quase $95 \%$ de cobertura de saneamento básico, sendo que a água potável chegou a $100 \%{ }^{17}$. Todos os indicadores apontam para uma melhora no bem estar da população, provavelmente contribuindo para esta tendência secular de crescimento positiva.

Neto e Filho ${ }^{18}$ ao buscarem informações que permitissem traçar um panorama sobre a tendência secular de crescimento desde o inicio do século XX, nos principais arquivos históricos brasileiros, comparando as medidas de comprimento-estatura de crianças de 1 a 12 anos, desde 1912 até 1996, afirmaram que do ponto de vista auxológico, ocorreu uma ten 
dência secular positiva do crescimento, mostrando a importância das transformações econômicas e sociais ocorridas.

Alguns dos estudos realizados para estimar a tendência secular de crescimento, concluem que esta apresenta uma tendência positiva a partir da segunda metade do século XX; coincidentemente, época de maior desenvolvimento econômico e maior bem estar social $^{19,20}$. Em 2000, Monteiro e Conde ${ }^{15}$, ao caracterizarem o crescimento linear nos primeiros cinco anos de vida, através de dados coletados por três inquéritos domiciliares realizados na cidade de São Paulo, SP, em 1974/75, 1984/85 e 1995/96, e demonstraram que mudanças positivas em determinantes distais do crescimento infantil como poder aquisitivo das famílias e escolaridade das mães, exerceu influência decisiva sobre a evolução favorável da altura das crianças.

Em nosso meio existem poucos estudos sobre tendência de crescimento, o que dificulta comparações, principalmente por causa das diferenças metodológicas, pois a maioria dos estudos é realizado em populações de crianças em idade escolar ou em adultos (jovens recrutados pelo serviço militar), mostrando quase sempre apenas a tendência em relação à estatura. Apesar das diferenças metodológicas, a maioria dos aqui apresentados mostrou tendência de crescimento positiva, que foi relacionada, do ponto de vista de causalidade com a melhoria nas condições de vida da população estudada.

A coexistência de uma maior prevalência de magreza e de excesso de peso descrito pelo IMC dos pré-escolares de 2007 de Taubaté, indicando também uma maior dispersão dos valores observados, é provavelmente decorrente de um ganho desproporcional entre peso e estatura dessas crianças, que resulta também na maior variabilidade observada no desvio padrão do IMC de 2007.
Contrariando a imagem positiva de crescimento de estatura, o aumento de peso, quando muito intenso, pode não ser um crescimento adequado, contribuindo para o aumento da prevalência de excesso de peso, que nas ultimas décadas vem ocorrendo em vários países, com o consequente aumentando no risco de doenças crônicas e degenerativas.

É incontestável que o Brasil e diversos países da América Latina nos últimos 20 anos estão passando por uma rápida transição demográfica, epidemiológica e nutricional ${ }^{21,22}$.

$\mathrm{O}$ fato de este estudo apresentar um aumento de prevalência de excesso de peso mostra que a transição nutricional, fenômeno no qual ocorre uma inversão nos padrões de distribuição dos problemas nutricionais de uma população, passando de uma condição de prevalência de desnutrição para uma de obesidade ${ }^{23,24}$, também está ocorrendo fora das grandes regiões metropolitanas, como a cidade de Taubaté.

Finalizando, é possível concluir que nos pré-escolares da cidade de Taubaté houve tendência secular de crescimento bastante significativa entre 1997 e 2007, mais acentuada para estatura do que para o peso. Junto com esta tendência, observou-se também um aumento na prevalência de crianças emagrecidas e, principalmente, com excesso de peso, o que confirma que simultaneamente ocorreu um importante processo de transição nutricional.

Isso sugere que, possivelmente, a melhoria das condições de bem estar da população, alem de repercutir positivamente no crescimento em estatura dos pré-escolares, também pode resultar na instalação de um intenso e precoce processo de transição nutricional, inclusive fora das grandes regiões metropolitanas. Se tendências como estas forem frequentemente observadas em diferentes populações, tornar-se-á necessário considerar as implicações que devem ter para a segurança e a vigilância alimentar e nutricional. 


\section{REFERÊNCIAS}

1. Forattini OP. Ecologia, epidemiologia e sociedade. São Paulo: EDUSP/Artes Médicas; 1992.

2. Jordan J, Ruben JM, Hernandez J, Bebelagua A, Tanner JM, Goldstein. The 1972 Cuban national child growth study as an example of population health monitoring: design and methods. Annals of Human Biology. 1975; 2(2): 153-171

3. Whitehead RG. Growth in weight and length. Acta Pediatrica. 2003; 92:406-410.

4. World Health Organization. Physical status: the use and interpretation of anthropometry. Geneva: WHO; 1995. (WHOTechnical Report Series, 854).

5. Cole TJ. The secular trend in human physical growth: a biological view. Economics and Human Biology. 2003; 1: 161-168.

6. Ali AMD, Uetake T, Ohtsuki F. Secular changes in relative leg length in postwar Japan. Am J Hum Biol. 2000; 12:405-416.

7. Loesch DZ, Strokes K, Huggins RM. Secular trend in body height and weight of Australian children and adolescents. Am J Phys Anthropol. 2000; 111:546-556.

8. Tanner JM. The potencial of auxological data for monitoring economic and social well-being. Social Science History.1982; 6:571-581.

9. Kac G. Tendência secular em estatura: uma revisão da literatura. Cad. Saúde Pública. 1999; 15(3):451-461

10. Zhang Y, Wang S. Secular trends in growth and body proportion among children and adolescents from 1985 to 2005 in Shandong, China. Anthropological Science. 2009; 117(2): 69-76.
11. Lohman TG, Roche AF, Matorell R. Antropometric standardization reference manual. 1988 Ilinois: Human Kinetics Publishers.

12. Leone C, Primo E, Castiglione MSG, Santos MG, Santos JM, Mascaretti LAS. Nutritional anthropometry of low socioeconomic level schoolchildren. Tema livre apresentado no $2^{\text {nd }}$ Internacional Congress of Nutrition in Pediatrics, promovido pela International Society of Pediatric Nutrition e Sociedade Portuguesa de Pediatria. Lisboa. Portugal. 1994.

13. Kuczmarski RJ, Ogden CL, Grummer SLM et al. CDC Growth Charts: United States-methods and development. National Center for Health Statistics. Vital Health Statistics.2000:246.

14. Monteiro CA. Critérios antropométricos no diagnóstico da desnutrição em programas de assistência a criança. Rev Saúde Pública. 1984;18(3):209-17.

15. World Health Organization. Software for assessing growth of the world's children and adolescents. Who Antro Plus for Personal Computers Manual. 2009.

16. Marmo DB, Zambom MP, Morcillo AM, Guimarey LM. Tendência secular de crescimento em escolares de Paulínia, São Paulo- Brasil (1979/80-1993/94). Rev Assoc Med Bras. 2004; 50(4):386-90.

17. Seade. Fundação Sistema Estadual de Análise de Dados. www.Seade.gov.br.2009.

18. Neto JE, Filho AAB. Tendência secular de crescimento em crianças do Brasil: evidencias de evolução positiva desde a primeira metade do século XX. Rev Cienc. Méd. 2004; 13(2):95-104. 
19. Monteiro CA, Torres AM. Can secular trends in child in growth be estimated from a single cross sectional survey? BMJ.1992; 305:797-9.

20. Monteiro CA, Benicio MH, Gouveia NC. Secular trends in Brazil over three decades. Ann Hum Biol. 1994; 21:381-90.

21. Monteiro CA, Conde WL. Tendência secular do crescimento pós-natal na cidade de São Paulo (1974-1996). Rev Saúde Pública. 2000; 34(6): 41-51.
22. Popkin BM. The nutrition transition and its health implications in lower-income countries. Public Health Nutr. 1998; 1:5-22.

23. Uauy R, Albala C, Kain J. Obesity trends in Latin America: transiting from under to overweight. J Nutr. 2001; 131:893S-99S.

24. Kac G. A transição nutricional e a epidemiologia da obesidade na América Latina. Cad Saúde Pública. 2003; 19 (Sup. 1) S4-S5.

Recebido em 02 de março de 2010 Modificado em 22 de abril de 2010 Aceito em 28 de maio de 2010 